\title{
Implementation of the Eclipse Process Framework Composer Tool for the Documentation of Quality Management Systems: A Case Applied in Healthcare Services
}

Juan-David De-la-Hoz-Hernández, Alexander Troncoso-Palacio, Emiro De-la-Hoz-Franco

\section{Abstract}

This document presents the implementation of the Eclipse Process Framework Composer EPFC tool, in a company in the health sector, which where it were using physical documentation to register the processes QMS Quality Management Systems, in this one it is explained the process of adapting the tool, to migrate the documentary structure of the company, through the application of a model that correlates the attributes of the tool with the documentary structure. The proposed model allows the construction of document structures for the QMS that guide the user in their processes and procedures within the organization. Therefore, it is proposed that by promoting knowledge management and reducing the execution time of the processes, it will be possible for companies to be more productive, improve customer service, failure rates in the development of activities be minimized and, and reduces the lead time in the processes. Finally, it was obtained a documentary platform developed under a process approach that implements interactive diagrams that aim to facilitate the understanding of each of the elements that make up the QMS.

Keywords:

Quality Management System - QMS, Eclipse Process Framework Composer - EPFC, Document Management System - DMS, Delays in documentation 